\title{
Effects of the Radius of Curvature on Natural Frequency and Mode Shape
}

\author{
Can Gonenli $^{1 *}$ Hasan Ozturk $^{2}$ and Oguzhan Das ${ }^{3}$ \\ ${ }^{1-3}$ The Graduate School of Natural and Applied Sciences, Dokuz Eylül University, İzmir, Turkey \\ ${ }^{2}$ Department of Mechanical Engineering, Dokuz Eylül University, İzmir, Turkey
}

\begin{abstract}
In this study, the effect of radius of curvature on the natural frequency of isotropic square thin plate is investigated. The models examined are obtained by simply changing the radius of curvature by keeping the length constant from the flat plate to the semicircle model. The free vibration analysis of the plates fixed on two straight edges is performed by the finite element method. As a finite element type, the four-node quadrilateral rectangular element type, which has a total of twenty four generalized coordinates, is used. Out-of-plane theory and in-plane theory are used together to form the curve model. The accuracy and validity of the theory are controlled with the data obtained from the finite element program. The effect of the curvature radius on the first five natural frequencies and the mode shapes of these natural frequencies is given in tables and graphs.
\end{abstract}

Key Words: Curve Plate, Finite Element, Mode Shape, Natural Frequency, Vibration

\section{Introduction}

Flat and curved plates with different geometries are used as structural elements in many engineering fields. Different theories of plates for a cylindrical structure are also the subject of different studies. Raffo and Quintana propose a general algorithm to obtain approximate analytical solutions for the study of the free vibrations of a rectangular anisotropic thin plate with an internal curved line hinge and general restraints [1]. Wong et al. studied with free vibration characteristics and natural frequency of horizontally curved plate girder bridges [2]. Hinton et al. studied free vibration analysis of prismatic folded plate and shell structures supported on diaphragms at two opposite edges with the other two edges arbitrarily restrained for curved, variable thickness finite strips based on Mindlin-Reissner shell theory [3]. Morris and Dave presented the finite strip method which is applied to the prediction of the natural frequencies of vibration of longitudinally invariant, rigidly connected assemblages of circularly curved and flat (zero curvature) strips having diaphragm end supports [4]. Belalia investigated the vibration behavior using a curved hierarchical finite element for sandwich elliptic plates [5]. Sandwich plates and cylindrical shells composed of two composite laminated faces and an ideally orthotropic elastic core are considered in the paper of Hwu et al. [6]. Shi et al. studied the dynamic prediction model of an annular plate-cylindrical shell structure with complex boundary conditions and coupling conditions is constructed [7]. 2D numerical approaches which are the generalized differential quadrature and the finite element methods are compared by Tornabene et al. with an exact 3D shell solution in the case of free vibrations of functionally graded material plates and shells [8]. Xie et al. studied free and forced vibrations of elastically coupled thin annular plate and cylindrical shell structures under elastic boundary conditions through wave based method [9]. Mercan et al. investigated the free vibration response of circular cylindrical shells with functionally graded material [10]. Yongqiang and Jian presented a curve strip Fourier p-element for free vibration analysis of circular and annular sectorial thin plates [11].

It is well-known knowledge that the stiffness of the plates increases with increasing the curvature. This information is used in bridge models, dam structures, and vehicle designs. However, the mode shapes that change with the change of curvature should also be considered as a design parameter. Furthermore, the continued increase in curvature sharply reduces the stiffness of the geometry. In this study, the natural frequency analysis of the fixed-length isotropic thin square plate, starting from the flat plate model to the 
semicircle model fixed on both edges, is performed. The results are confirmed by the finite element program. In addition, the mode shapes affected by the first five natural frequency changes are shown.

\section{Mathematical Modeling of Plate}

To create flat and curved plate models, the four-node quadrilateral element is selected for using the finite element method. While making this selection it is known that to create the curvilinear design, the plate must be rotatable around any of its axes. The selected model of finite elements must therefore also contain six generalized coordinates in each node. To reach this finite element model, out-of-plane theory and in-plane theory are used together.

\subsection{Flexural Vibration of Plates}

The principle of flexural vibration is based on the out-of-plane theory. The finite element model to be used for the form of bending motion must have a minimum of twelve corner displacements corresponding to three generalized coordinates at each corner, $w, d w / d y$ and $d w / d x$, and this finite element model as shown in Figure-1.

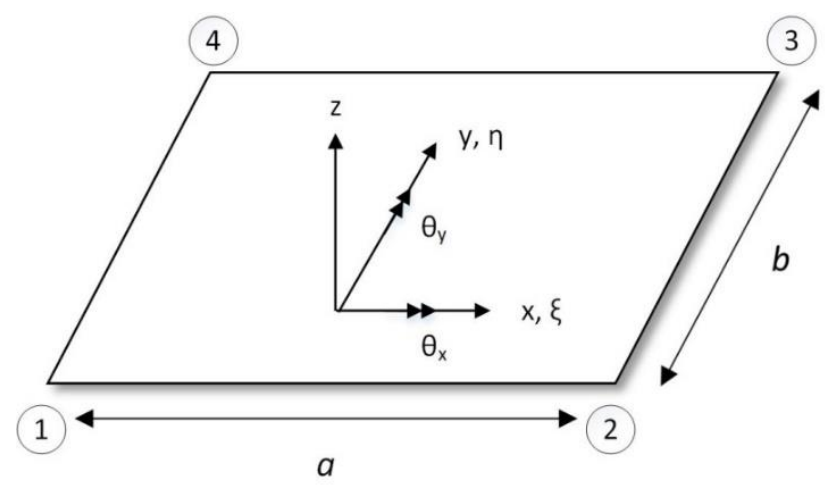

Figure 1. Degrees of freedom for the quadrilateral rectangular plate model for out-of-plane vibration.

In equation (1), the shape function is given for the flexural vibration of plate [12].

$$
N_{j}^{T}(\xi, \eta)=\left[\begin{array}{c}
\frac{1}{8}\left(1+\xi_{j} \xi\right)\left(1+\eta_{j} \eta\right)\left(2+\xi_{j} \xi+\eta_{j} \eta-\xi^{2}-\eta^{2}\right) \\
\frac{b}{2}\left(1+\xi_{j} \xi\right)\left(\eta_{j}+\eta\right)\left(\eta^{2}-1\right) \\
-\frac{a}{2}\left(\xi_{j}+\xi\right)\left(\xi^{2}-1\right)\left(1+\eta_{j} \eta\right)
\end{array}\right]
$$

$(\xi j, \eta j)$ are the coordinates of node $j$. Element stiffness matrix $[K]_{1}$ is calculated and is given in equation (2).

$$
[K]_{1}=\int_{-1}^{1} \int_{-1}^{1}[B]^{T}[D][B] \mathrm{d} \xi \mathrm{d} \eta
$$

Where $D$ is a material matrix for flexural vibration and given in equation (3).

$$
[D]=\frac{E h^{3}}{12\left(1-v^{2}\right)}\left[\begin{array}{ccc}
1 & v & 0 \\
v & 1 & 0 \\
0 & 0 & \frac{1-v}{2}
\end{array}\right]
$$

$E$ represents Young modulus, $h$ represents thickness of the plate and $v$ represents Poisson's ratio. The strain displacement matrix $B$ is obtained by equation (4). 


$$
B=\left[\begin{array}{c}
\frac{4}{(a)^{2}} \frac{\partial^{2}}{\partial \xi^{2}} \\
\frac{4}{(b)^{2}} \frac{\partial^{2}}{\partial \eta^{2}} \\
\frac{8}{(a)(b)} \frac{\partial^{2}}{\partial \xi \partial \eta}
\end{array}\right] N(\xi, \eta)
$$

The mass matrix is also calculated using the equation (5).

$$
[M]_{1}=\rho h \int_{-1}^{1} \int_{-1}^{1}[N]^{T}[N] \mathrm{d} \xi \mathrm{d} \eta
$$

Where $\rho$ represents the density of the material.

\subsection{In-Plane Vibration of Plates}

The finite element model to be used for the in-plane vibration form must have eight displacements at each corner including $u, v$ and $\theta_{z}$, and this finite element model is shown in Figure-2.

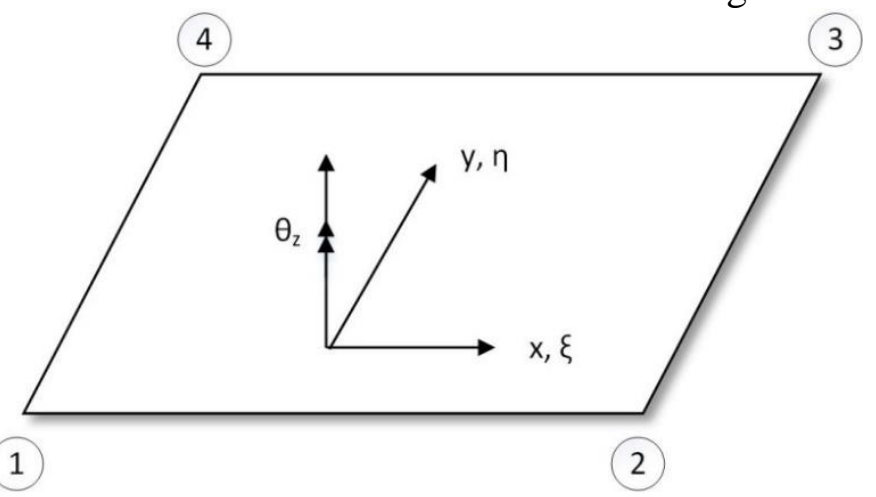

Figure 2. Degrees of freedom for the quadrilateral rectangular plate model for in-plane vibration.

The in-plane theory shape function is given in equation $(6)[12,13]$.

$$
N_{j}(\xi, \eta)=\frac{1}{4}\left(1+\xi_{j} \xi\right)\left(1+\eta_{j} \eta\right)
$$

The components $u$ and $v$ of the element displacement are given in equation (7).

$$
\begin{aligned}
& u=N_{1} q_{1}+N_{2} q_{3}+N_{3} q_{5}+N_{4} q_{7} \\
& v=N_{1} q_{2}+N_{2} q_{4}+N_{3} q_{6}+N_{4} q_{8}
\end{aligned}
$$

Where $q_{i}(i=1$ to 8$)$ represents the denotes the element displacement vector The strain-displacement relations are:

$$
\varepsilon=\left\{\begin{array}{c}
\varepsilon_{x} \\
\varepsilon_{y} \\
\Upsilon_{x y}
\end{array}\right\}=\left\{\begin{array}{c}
\frac{\partial u}{\partial x} \\
\frac{\partial u}{\partial y} \\
\frac{\partial u}{\partial y}+\frac{\partial v}{\partial x}
\end{array}\right\}
$$

Subsequently, it needs to express the derivatives of the displacement functions, which are in $x$ and $y$ coordinates, in terms of its derivatives in $\xi, \eta$ coordinates. 


$$
\begin{aligned}
& \left\{\begin{array}{l}
\frac{\partial u}{\partial \xi} \\
\frac{\partial u}{\partial \eta}
\end{array}\right\}=J\left\{\begin{array}{l}
\frac{\partial u}{\partial x} \\
\frac{\partial u}{\partial y}
\end{array}\right\} \\
& \left\{\begin{array}{l}
\frac{\partial v}{\partial \xi} \\
\frac{\partial v}{\partial \eta}
\end{array}\right\}=J\left\{\begin{array}{l}
\frac{\partial v}{\partial x} \\
\frac{\partial v}{\partial y}
\end{array}\right\}
\end{aligned}
$$

Where $\mathrm{J}$ is the Jacobian matrix.

$$
J=\left[\begin{array}{ll}
\frac{\partial x}{\partial \xi} & \frac{\partial y}{\partial \xi} \\
\frac{\partial x}{\partial \eta} & \frac{\partial y}{\partial \eta}
\end{array}\right]
$$

Equation (8) now yield:

$$
\varepsilon=A\left\{\begin{array}{l}
\frac{\partial u}{\partial \xi} \\
\frac{\partial u}{\partial \eta} \\
\frac{\partial v}{\partial \xi} \\
\frac{\partial v}{\partial \eta}
\end{array}\right\}
$$

where $\mathrm{A}$ is given by:

$$
A=\frac{1}{\operatorname{det} J}\left[\begin{array}{cccc}
J_{22} & -J_{12} & 0 & 0 \\
0 & 0 & -J_{21} & J_{11} \\
-J_{21} & J_{11} & J_{22} & -J_{12}
\end{array}\right]
$$

From the interpolation equations, equation (13) is obtained:

$$
\left\{\begin{array}{l}
\frac{\partial u}{\partial \xi} \\
\frac{\partial u}{\partial \eta} \\
\frac{\partial v}{\partial \xi} \\
\frac{\partial v}{\partial \eta}
\end{array}\right\}=G q
$$

By substituting equation (13) into (11), strain displacement matrix is obtained.

$$
B=A G
$$

Element stiffness matrix $[K]_{2}$ is calculated through equation (15).

$$
[K]_{2}=h \int_{-1}^{1} \int_{-1}^{1} B^{T} D B \operatorname{det} J d \xi d \eta
$$

Where $D$ is a material matrix for in-plane vibration. 


$$
[D]=\frac{E}{\left(1-v^{2}\right)}\left[\begin{array}{ccc}
1 & v & 0 \\
v & 1 & 0 \\
0 & 0 & \frac{1-v}{2}
\end{array}\right]
$$

The element mass matrix is also given in equation (17).

$$
[M]_{2}=\rho h \int_{-1}^{1} \int_{-1}^{1} N^{T} N \operatorname{det} J d \xi d \eta
$$

\subsection{Finite Element Model}

The stiffness and the mass matrices of the finite element model are obtained by combining the out-of-plane theory and the in-plane theory. The relationship is defined in equation (18).

$$
\text { [Out of plane, } \left.K_{1} \& M_{1}\right]_{3 \times 3}+\left[\text { In plane, } K_{2} \& M_{2}\right]_{2 \times 2}=\left[\begin{array}{cc}
3 \times 3 & 0 \\
0 & 2 \times 2
\end{array}\right]_{5 \times 5}
$$

This model has six degrees of freedom, but it has matrices of stiffness and mass $(5 \times 5)$ size. Although these matrices have the effect of $\theta_{z}$, the last value of the drilling effect must be formally applied to the matrices. In equation (19), this relationship is given.

$$
K_{e}, M_{e}=\left[\begin{array}{cc}
3 \times 3 & 0 \\
0 & 2 \times 2
\end{array}\right]_{5 \times 5}+\theta_{z}=\left[\begin{array}{ccc}
3 \times 3 & 0 & 0 \\
0 & 2 \times 2 & 0 \\
0 & 0 & 1 \times 1
\end{array}\right]_{6 \times 6}
$$

The value of $\theta_{z}$ taken the absolute value that $1 / 1000$ of the minimum value in $(5 \times 5)$ stiffness and mass matrices [14]. After adding, the mathematically obtained rectangular shell element model with six degrees of freedom is given in Figure-3.

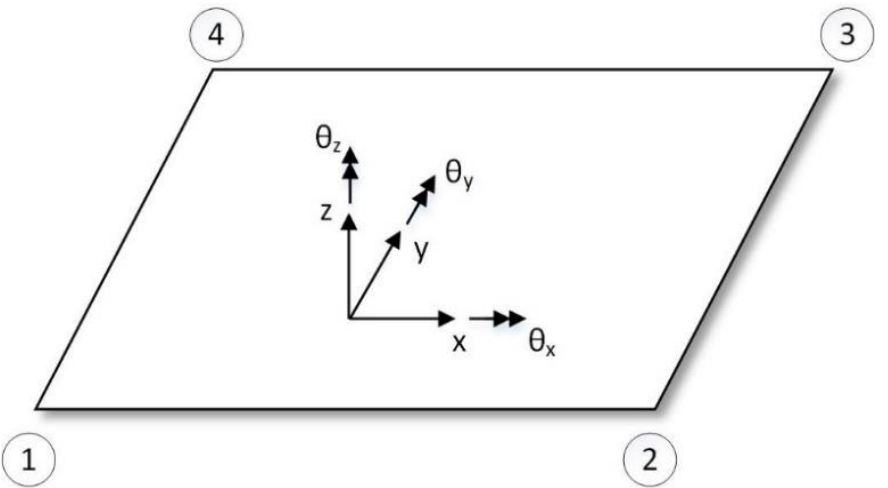

Figure 3. The quadrilateral rectangular straight plate element with six degrees of freedom.

\subsection{Curve Model}

To examine the effect of the radius of curvature, firstly the flat plate fixed on both sides. At this point, the radius of curvature is theoretically infinite. For the transition to the curved plate, the radius of curvature is then reduced from infinite to $160 \mathrm{~mm}$, where the structure is exactly a semicircle. The length of the plate is kept constant so that the natural frequency change can be seen during the radius change. Figure-4 shows a linear representation of curved plate models ranging from flat model to semicircle. 


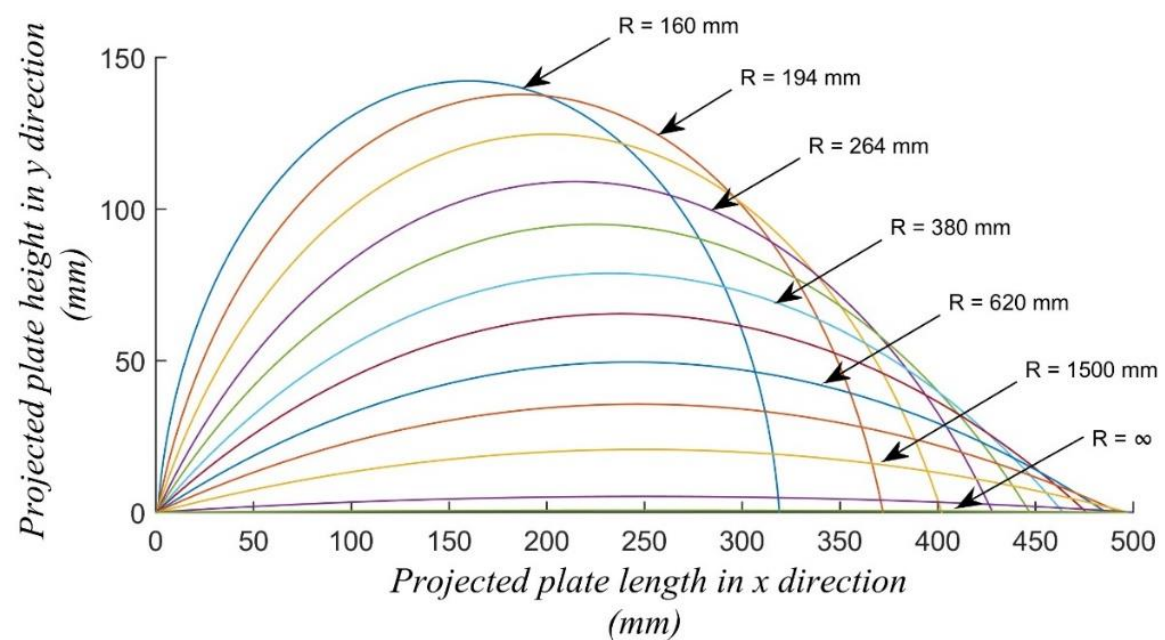

Figure 4. Changing of flat model to semicircle model with constant length.

To obtain these curve models with flat plate, the flat plate finite element model is rotated about the y axis. Rotation of the coordinate system is given in Figure-5.

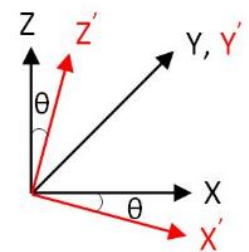

(a)



(b)

Figure 5. Rotation of the coordinate system. (a) the rotation of the axes of translation, (b) the rotation of the axes of rotation.

The transformation matrix used to perform the rotation mathematically, is given in Table-1.

Table 1. Transformation matrix.

\begin{tabular}{|c|c|c|c|c|c|c|}
\hline $\boldsymbol{\theta}_{\mathbf{x}}$ & $\boldsymbol{\theta}_{\mathbf{y}}$ & $\mathbf{w}$ & $\mathbf{u}$ & $\mathbf{v}$ & $\boldsymbol{\theta}_{\mathbf{z}}$ & \\
\hline $\cos (\theta)$ & 0 & 0 & 0 & 0 & $\sin (\theta)$ & $\boldsymbol{\theta}_{\mathbf{x}}$ \\
\hline 0 & 1 & 0 & 0 & 0 & 0 & $\boldsymbol{\theta}_{\mathbf{y}}$ \\
\hline 0 & 0 & $\cos (\theta)$ & $-\sin (\theta)$ & 0 & 0 & $\mathbf{w}$ \\
\hline 0 & 0 & $\sin (\theta)$ & $\cos (\theta)$ & 0 & 0 & $\mathbf{u}$ \\
\hline 0 & 0 & 0 & 0 & 1 & 0 & $\mathbf{v}$ \\
\hline$-\sin (\theta)$ & 0 & 0 & 0 & 0 & $\cos (\theta)$ & $\boldsymbol{\theta}_{\mathbf{z}}$ \\
\hline
\end{tabular}

The stiffness and mass matrices can be obtained through the transformation matrix is given in equation (20).

$$
\begin{aligned}
& K_{r}=T^{T} \times K_{e} \times T \\
& M_{r}=T^{T} \times M_{e} \times T
\end{aligned}
$$

Where $k_{e}$ and $m_{e}$ represent stiffness and mass matrices of the reference plate. Then rotated matrices $K_{r}$ and $M_{r}$ can be obtained with the multiplication of transformation and reference matrices. Stiffness matrix $K_{e}$ and mass matrix $M_{e}$ of each plate element are used to form global stiffness $(K)$ and global mass $(M)$ matrices. The dynamic response of a plate for the global system can be formulated via Lagrange's equation of motion (21). 


$$
[M]\{\ddot{q}\}+[[K]]\{q\}=0
$$

Natural frequencies can be obtained via equation (22), where $\lambda$ includes natural frequency parameter $\omega^{2}$.

$$
(K)-\lambda M=0
$$

\section{Results}

The geometric and material properties are: $E=200 \mathrm{GPa}, \rho=7870 \mathrm{~kg} / \mathrm{m}^{3}, v=0.29 ; a=0.5 \mathrm{~m}, b=0.5 \mathrm{~m}$, and $h=5$ $\mathrm{mm}$. The effects of radius of curvature on the natural frequency, $\lambda$ and mode shapes for the first five modes are investigated. $\lambda$ is given in equation (23).

$$
\lambda=\omega \sqrt{\frac{\rho A a^{4}}{E I}}
$$

To verify the reliability and validity of the present model, the natural frequency parameters obtained from this study are compared with the results obtained from ANSYS software. Representative flat and semicircle models with two sides fixed are shown in Figure-6. Table-2 shows a comparison of the results between the present study and ANSYS.

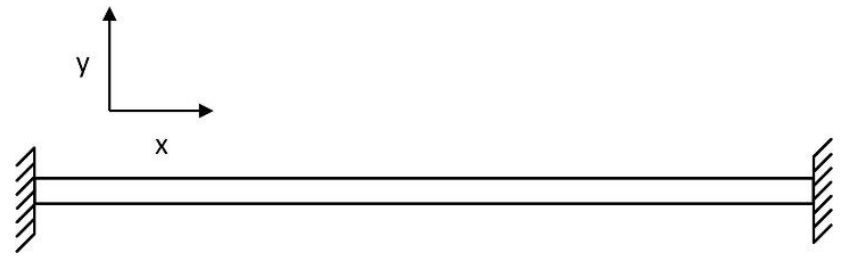

(a)

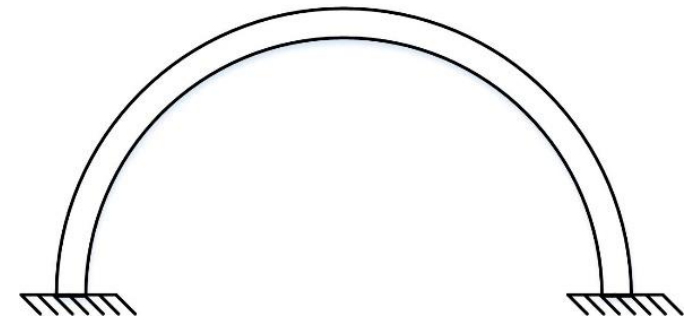

(b)

\begin{tabular}{|c|c|c|c|c|c|c|c|c|c|c|}
\hline \multirow{2}{*}{$\begin{array}{c}\text { Radius } \\
(\mathrm{mm})\end{array}$} & \multicolumn{2}{|c|}{$\lambda_{1}$} & \multicolumn{2}{|c|}{$\lambda_{2}$} & \multicolumn{2}{|c|}{$\lambda_{3}$} & \multicolumn{2}{|c|}{$\lambda_{4}$} & \multicolumn{2}{|c|}{$\lambda_{5}$} \\
\hline & ANSYS & Present & ANSYS & Present & ANSYS & Present & ANSYS & Present & ANSYS & Present \\
\hline Flat & 3.70 & 3.69 & 4.41 & 4.40 & 7.25 & 7.25 & 10.18 & 10.19 & 11.18 & 11.19 \\
\hline 5000 & 5.90 & 5.95 & 6.37 & 6.41 & 8.77 & 8.76 & 10.18 & 10.21 & 11.32 & 11.35 \\
\hline 4500 & 6.30 & 6.35 & 6.74 & 6.79 & 9.08 & 9.08 & 10.18 & 10.21 & 11.36 & 11.39 \\
\hline 4000 & 6.81 & 6.88 & 7.23 & 7.29 & 9.51 & 9.52 & 10.17 & 10.21 & 11.40 & 11.44 \\
\hline 3500 & 7.49 & 7.57 & 7.88 & 7.95 & 10.09 & 10.11 & 10.17 & 10.21 & 11.47 & 11.51 \\
\hline 3000 & 8.42 & 8.51 & 8.77 & 8.86 & 10.17 & 10.21 & 10.93 & 10.96 & 11.58 & 11.62 \\
\hline 2500 & 9.72 & 9.83 & 10.04 & 10.15 & 10.17 & 10.20 & 11.75 & 11.79 & 12.18 & 12.23 \\
\hline 2000 & 10.17 & 10.19 & 11.60 & 11.73 & 11.93 & 12.06 & 12.06 & 12.11 & 14.15 & 14.22 \\
\hline 1500 & 10.15 & 10.18 & 12.68 & 12.76 & 14.28 & 14.42 & 14.72 & 14.87 & 17.49 & 17.57 \\
\hline 1000 & 10.10 & 10.12 & 14.25 & 14.40 & 16.91 & 17.02 & 17.73 & 17.84 & 21.58 & 21.71 \\
\hline 500 & 9.80 & 9.82 & 17.83 & 17.91 & 18.76 & 18.85 & 19.69 & 20.00 & 29.10 & 29.23 \\
\hline 160 & 7.18 & 7.22 & 15.75 & 15.88 & 15.92 & 16.08 & 25.36 & 25.73 & 29.20 & 29.58 \\
\hline
\end{tabular}

Figure 6. Plate models. (a) flat plate model, (b) semicircle plate model.

Table 2. Comparison results between present model and ANSYS model.

Table-2 shows that the natural frequency parameter results of the present model and the error rates respect to ANSYS results are quite consistent. The results of the first five natural frequency parameters of the curved plate model starting from $5000 \mathrm{~mm}$ radius until the semicircle changes are given in Figure-7. 


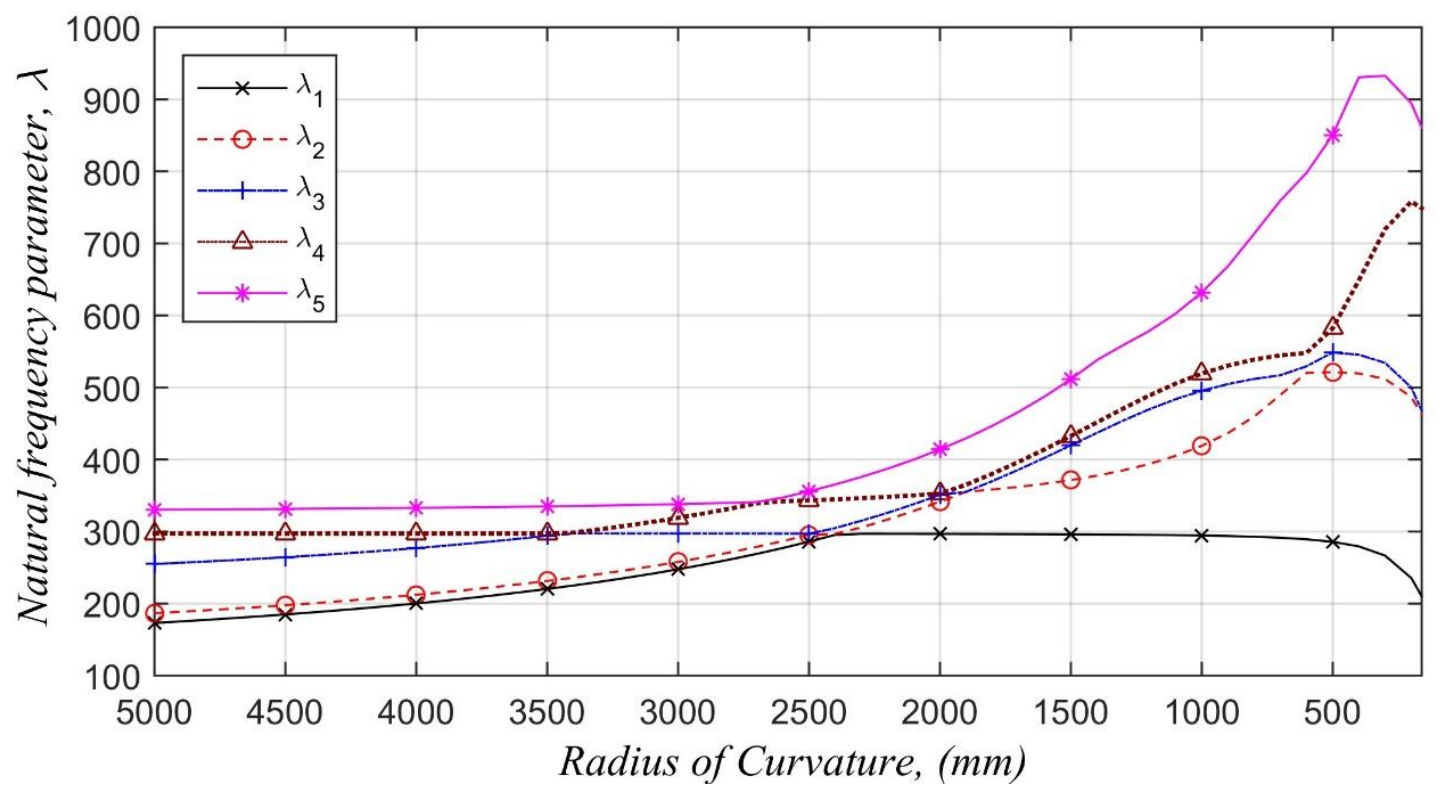

Figure 7. Effect of the radius of curvature on the natural frequencies

When the results of the natural frequency parameters from the flat plate model to the semicircle plate model are examined, it is seen that from Figure-7, each natural frequency parameter curve changes its behavior at a different radius of curvature. Different mode shapes are observed with these natural frequency parameter curve changes. Moreover, each natural frequency parameter shows a sharp decrease when the radius of curvature is close to the semicircle.

When the mode shapes belong the first five natural frequencies are examined, the constant mode shape occurs up to a radius of curvature of $2400 \mathrm{~mm}$ for the first natural frequency parameter, and then a different mode shape occurs. The two different mode shapes are given in the Figure-8. Also, all mode shape graphs are given in the rectangular plate plane.

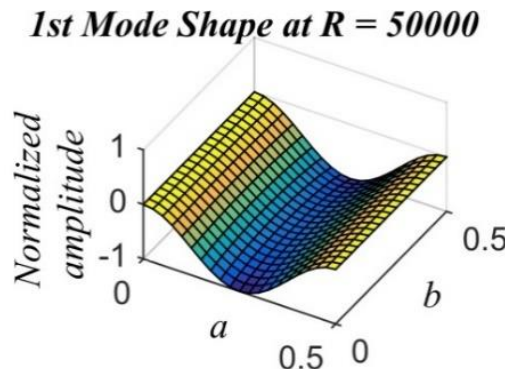

(a) 1st Mode Shape at $R=2200$

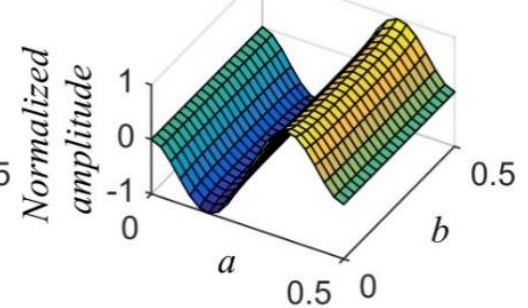

(b)

Figure 8. The two different mode shapes for the first natural frequency.

Figure 8-a represents the mode shape for the radius of curvature value between the flat plate and the 2400 $\mathrm{mm}$, Figure 8-b represents the mode shape in the section where the radius varies between $2400 \mathrm{~mm}$ and 160 $\mathrm{mm}$.

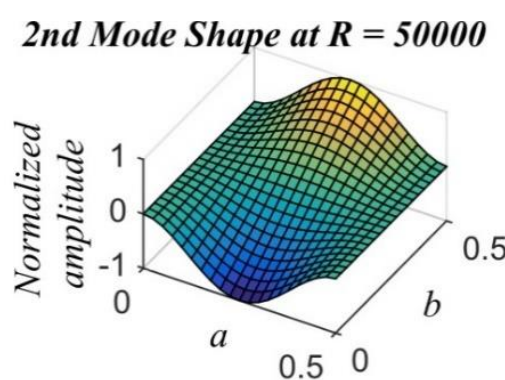

(a)

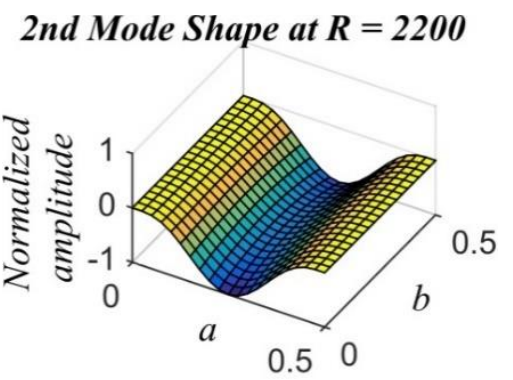

(b) 


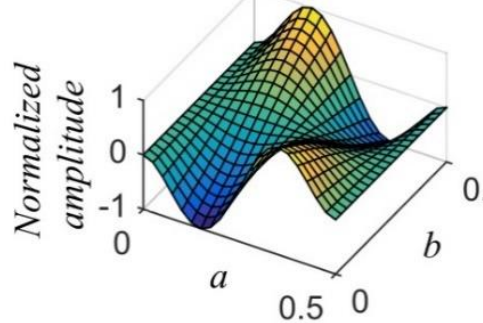

(c)

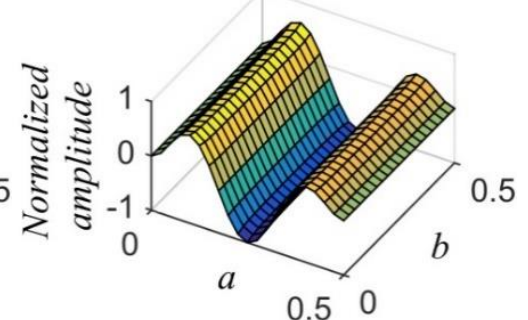

(d)

Figure 9. The four different mode shapes for the second natural frequency.

Figure 9-a represents the mode shape for the radius of curvature value between the flat plate and the 2500 $\mathrm{mm}$. Figure 9-b represents the mode shape for all radius of curvature in the range of 2500 to $2000 \mathrm{~mm}$, Figure 9-c represents the mode shape for all radius of curvature in the range of 2000 to $600 \mathrm{~mm}$, Figure 9-d represents the mode shape for all radius of curvature in the range of 600 to $160 \mathrm{~mm}$.

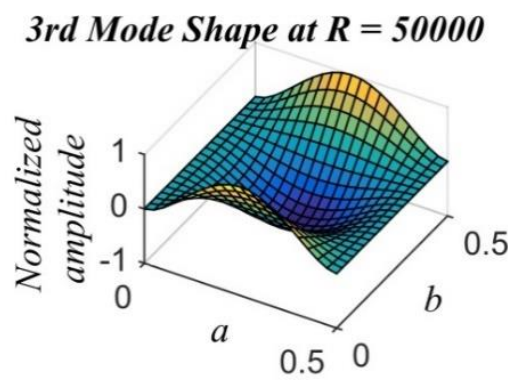

(a)

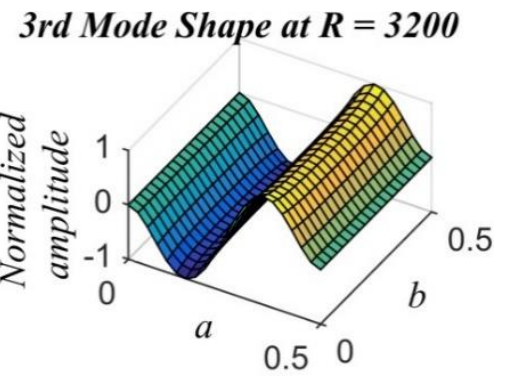

(b)

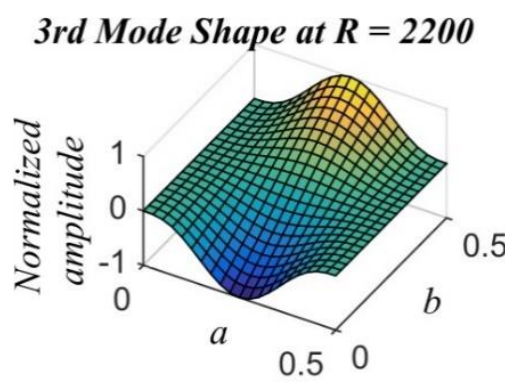

(c)

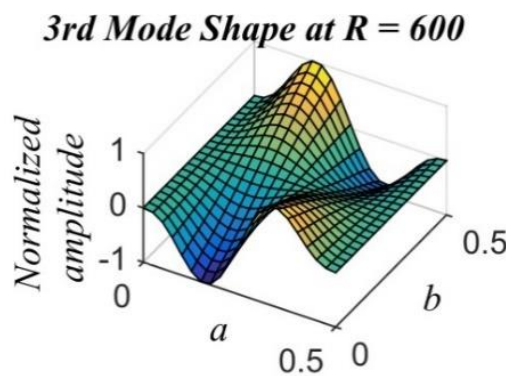

(e)

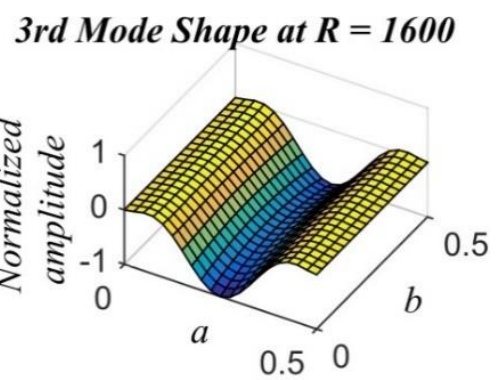

(d)

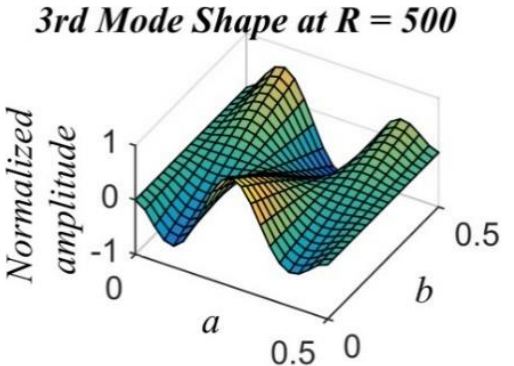

(f)

Figure 10. The six different mode shapes for the third natural frequency.

Figure 10-a represents the mode shape for the radius of curvature value between the flat plate and the 3500 $\mathrm{mm}$, Figure 10-b represents the mode shape for the radius of curvature in the range of 3500 to $2500 \mathrm{~mm}$, Figure 10-c represents the mode shape for the radius of curvature in the range of 2500 to $2000 \mathrm{~mm}$, Figure 10 - $d$ represents the mode shape for the radius of curvature in the range of 2000 to $800 \mathrm{~mm}$, Figure 10-e represents the mode shape for the radius of curvature in the range of 800 to $500 \mathrm{~mm}$, Figure 10 -f represents the mode shape for the radius of curvature in the range of 500 to $160 \mathrm{~mm}$. 


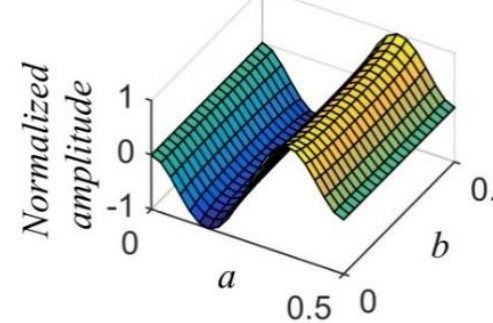

(a)

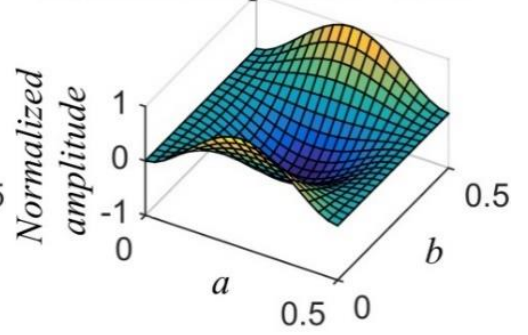

(b)

4th Mode Shape at $R=2200$

4th Mode Shape at $R=1600$

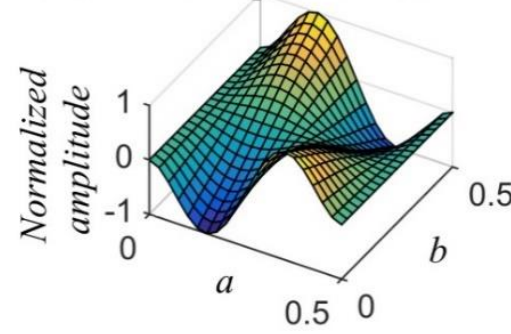

(c)

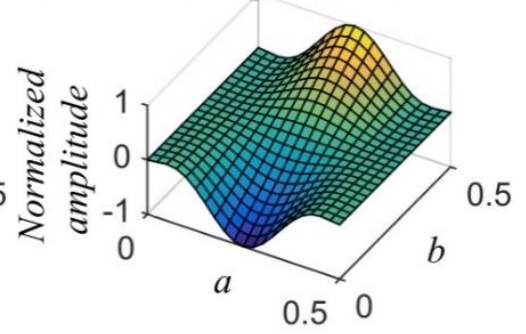

(d)

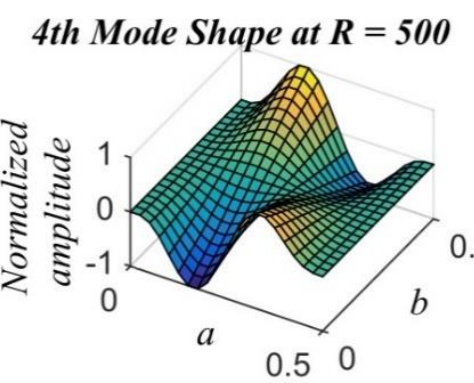

(e) 4th Mode Shape at $R=300$

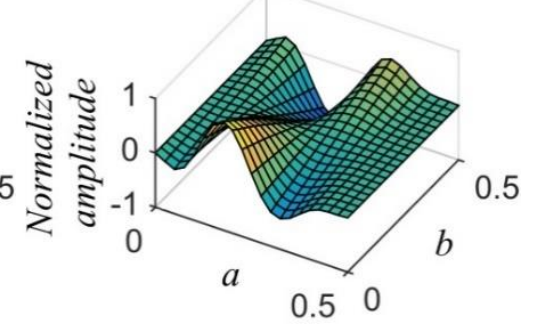

(f)

Figure 11. The six different mode shapes for the fourth natural frequency.

Figure 11-a represents the mode shape for the radius of curvature value between the flat plate and the 3500 $\mathrm{mm}$, Figure 11-b represents the mode shape for the radius of curvature in the range of 3500 to $2700 \mathrm{~mm}$, Figure 11-c represents the mode shape for the radius of curvature in the range of 2700 to $2000 \mathrm{~mm}$, Figure $11-\mathrm{d}$ represents the mode shape for all radius of curvature in the range of 2000 to $1600 \mathrm{~mm}$, Figure 11-e represents the mode shape for the radius of curvature in the range of 1600 to $600 \mathrm{~mm}$, Figure 11-f represents the mode shape for the radius of curvature in the range of 600 to $160 \mathrm{~mm}$.

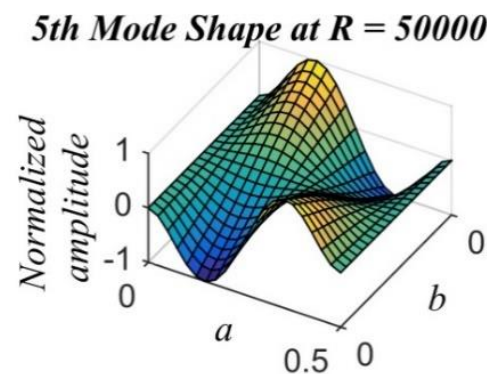

(a)

5th Mode Shape at $R=2200$

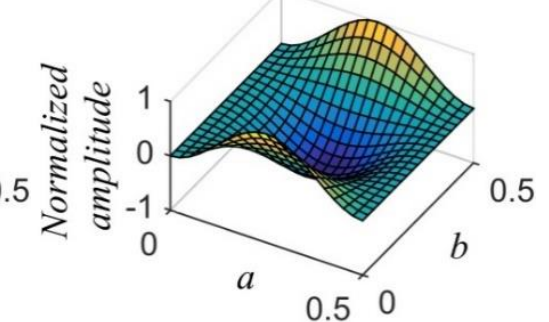

(b)

5th Mode Shape at $R=500$

5th Mode Shape at $R=300$

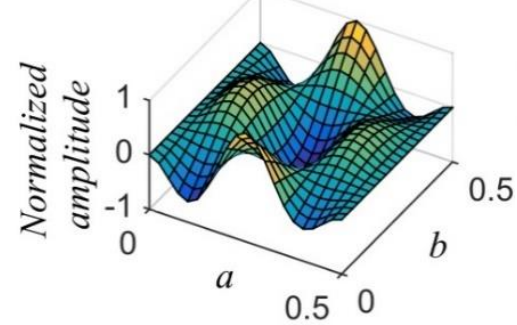

(c)

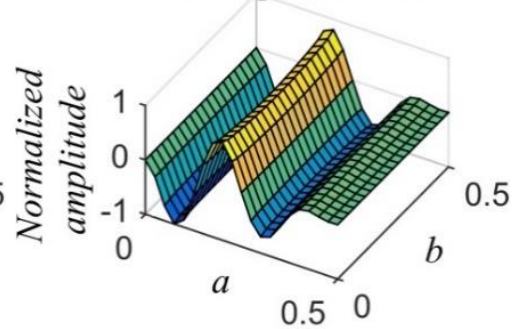

(d) 
Figure 12. The four different mode shapes for the fifth natural frequency.

Figure 12-a represents the fifth mode shape for the radius of curvature value between the flat plate and the $2700 \mathrm{~mm}$, Figure 12-b represents the fifth mode shape for the radius of curvature in the range of 2700 to $1400 \mathrm{~mm}$, Figure 12-c represents the fifth mode shape for the radius of curvature in the range of 1400 to 400 $\mathrm{mm}$, Figure 12-d represents the fifth mode shape for the radius of curvature in the range of 400 to $160 \mathrm{~mm}$. Figure 8-12 shows the mode shapes resulting from changes in the natural frequency parameter curves given in Figure 7. Consequently, different mode shapes occur at different points where the shape of the natural frequency parameter curvature changes. In addition, it is seen that the natural frequency parameter value shows a sudden decrease for all five natural frequencies where the geometry is closer to the semicircle.

\section{Results}

In this study, the effect of the radius of curvature on the natural frequency parameter is investigated by keeping the plate length constant. The first five natural frequencies are examined for all radius of curvature from the flat plate model to the semicircle plate model. Moreover, the mode shape changes due to the radius of curvature are also examined. According to the results obtained:

- As the curvature of the plate increases with constant length, the natural frequency values also increase to a certain level for all natural frequency parameters.

- By changing the radius of the curvature, more than one mode shape occurs for each natural frequency parameter.

- With the change of the radius of curvature, the natural frequency parameter curve behaves differently for each natural frequency parameter. Each change in the curves shows that a different mode shape occurs.

- As the radius of curvature moves the plate closer to the semicircle geometry, the increase in all natural frequency parameters stops, and then all values begin to decrease sharply.

\section{References}

[1] Raffo, J. L., \& Quintana, M. V. (2017). Natural vibrations of anisotropic plates with an internal curve with hinges. International Journal of Mechanical Sciences, 120, 301-310. doi: 10.1016/j.ijmecsci.2016.11.031

[2] Wong, M., Shanmugam, N., \& Osman, S. (2010). Free vibration characteristics of horizontally curved composite plate girder bridges. Steel \& Composite Structures, 10(4), 297-315. doi: 10.12989/scs.2010.10.4.297

[3] Hinton, E., \& Rao, N. (1993). Analysis and shape optimisation of variable thickness prismatic folded plates and curved shells - Part 1: Finite strip formulation. Thin-Walled Structures, 17(2), 81-111. doi: 10.1016/0263-8231(93)90029-a

[4] Morris, I., \& Dawe, D. (1980). Free vibration of curved-plate assemblies with diaphragm ends. Journal of Sound and Vibration, 73(1), 1-17. doi: 10.1016/0022-460x(80)90488-5

[5] Belalia, S. A. (2018). A curved hierarchical finite element method for the nonlinear vibration analysis of functionally graded sandwich elliptic plates. Mechanics of Advanced Materials and Structures, 26(13), 1115-1129. doi: 10.1080/15376494.2018.1430277

[6] Hwu, C., Hsu, H., \& Lin, Y. (2017). Free vibration of composite sandwich plates and cylindrical shells. Composite Structures, 171, 528-537. doi: 10.1016/j.compstruct.2017.03.042

[7] Shi, X.-J., Lİ, C.-L., \& Jiang, H.-B. (2018). Vibration characteristics of an annular plate-cylindrical shell structure with complex boundary conditions. Shock and Vibration, 31(1), 118-124. doi: 10.16385/j.cnki.issn.1004-4523.2018.01.014

[8] Tornabene, F., Brischetto, S., Fantuzzi, N., \& Viola, E. (2015). Numerical and exact models for free vibration analysis of cylindrical and spherical shell panels. Composites Part B: Engineering, 81, 231-250. doi: 10.1016/j.compositesb.2015.07.015

[9] Xie, K., Chen, M., Zhang, L., \& Xie, D. (2017). Wave based method for vibration analysis of elastically coupled annular plate and cylindrical shell structures. Applied Acoustics, 123, 107-122. doi: 10.1016/j.apacoust.2017.03.012 
[10] Mercan, K., Demir, Ç., \& Civalek, Ö. (2016). Vibration analysis of FG cylindrical shells with powerlaw index using discrete singular convolution technique. Curved and Layered Structures, 3(1). doi: 10.1515/cls-2016-0007

[11] Yongqiang, L., \& Jian, L. (2007). Free vibration analysis of circular and annular sectorial thin plates using curve strip Fourier p-element. Journal of Sound and Vibration, 305(3), 457-466. doi: 10.1016/j.jsv.2007.04.022

[12] Petyt, M. (2010). Introduction to finite element vibration analysis. New York, USA: Cambridge University Press

[13] Chandrupatla, T.R.; Belegundu, A.D. 2002. Introduction to Finite Elements in Engineering. New Jersey: Prentice Hall.

[14] Niyogi, A.G; Laha, M.K.; Sinha, P.K. Finite Element Vibration Analysis of Laminated Composite Folded Plate Structures; Shock and Vibration; 1999, Volume 6, Issue 5-6, pp.273-283, doi: $10.1155 / 1999 / 354234$ 